\title{
Treatment of Sporotrichosis With Itraconazole
}

\author{
Patricia Kay Sharkey-MathIS, M.D., San Antonio, Texas, Carol A. KaUfFMan, M.D., \\ Ann Arbor, Michigan, JOHN R. GRAYBILL, M.D., San Antonio, Texas, DAVID A. STEVENS, M.D., \\ JOHN S. HOSTETLER, M.D., San Jose, California, GRETCHEN CLOUD, M.S., \\ WILLIAM E. DISMUKES, M.D., Birmingham, Alabama, \\ and Other Members of the NIAID Mycoses Study Group*
}

PURPOSE: To describe the clinical presentation and outcomes of treatment with itraconazole in patients with sporotrichosis.

METHODS: A culture for Sporothrix schenckii or compatible histopathology was required for inclusion in the study. Patients with both cutaneous and systemic sporotrichosis were treated. Patients received from 100 to $600 \mathrm{mg}$ of itraconazole daily for 3 to 18 months. Patients were classified as responders or nonresponders. Responders were further classified as remaining on treatment, relapsed, or free of disease. Nonresponders included patients who failed to respond or progressed during treatment with itraconazole.

RESULTS: Twenty-seven patients (mean age: 53 years) were treated with 30 courses of itraconazole. Diabetes mellitus and alcoholism were present in eight and seven patients, respectively. Sites of involvement included lymphocutaneous alone in 9 patients, articular/osseous in 15 (multifocal in 3), and lung in 3. Prior therapy was unsuccessful in 11 patients. Among the 30 courses, there were 25 responders and 5 nonresponders. All 5 nonresponders received at least $200 \mathrm{mg}$ daily of itraconazole for durations that ranged from 6 to 18 months. Of the 25 responders, 7 relapsed 1 to 7 months after treatment durations of 6 to 18 months. Of the 7 who relapsed, 2 are responding to a second course. One responder was lost to follow-up after 10 months of

\footnotetext{
*See appendix.

From the Audie L. Murphy Memorial Veterans Hospital and University of Texas Health Science Center (PKS-M, JRG), San Antonio, Texas; the Department of Veterans Affairs Medical Center and University of Michigan Medical School (CAK), Ann Arbor, Michigan; the Santa Clara Valley Medical Center and Stanford University Medical School (DAS, JSH), San Jose, California; University of Alabama at Birmingham (GC, WED), Birmingham, Alabama, and the National Institute of Allergy and Infectious Diseases (members of the NIAID Mycoses Study Group), Bethesda, Maryland.

This work was supported in part by the National Institute of Allergy and Infectious Diseases Mycoses Study Group (Grant NOI-Al-52562) and Janssen Research Foundation.

Requests for reprints should be addressed to Patricia Kay SharkeyMathis, M.D., Department of Medicine, Division of Infectious Diseases, University of Texas Health Science Center, 7703 Floyd Curl Drive, San Antonio, Texas 78284-7881.

Manuscript submitted September 17, 1992, and accepted in revised form January 15, 1993.
}

treatment with itraconazole. Of the remaining 17 responders, 3 remain on treatment, and 14 are free of disease over follow-up durations of 6 to 42 months (mean: 17.6 months). Itraconazole was well tolerated with few side effects noted.

CONCLUSIONS: These results document the efficacy of itraconazole in the treatment of cutaneous and systemic sporotrichosis.

Q porotrichosis is a mycotic infection caused by the dimorphic fungus Sporothrix schenckii. The organism exists saprophytically in nature worldwide [1-7]. The spectrum of disease has been classified into two major categories: systemic and cutaneous inoculation sporotrichosis, with the latter considered far more common [1-6,8]. Involvement in systemic sporotrichosis may be multifocal or unifocal, typically with cutaneous, pulmonary, articular, osseous, genitourinary, or central nervous system foci [1-3]. Opportunistic infections may account for the increasing frequency of systemic sporotrichosis $[2,7,8]$. The poorer prognosis with multifocal systemic disease is probably due to the more severe underlying immunosuppression in these patients [2]. More common than multifocal disease, unifocal systemic sporotrichosis often has a chronic, progressive course $[4,8]$. A pulmonary portal of entry, with hematogenous dissemination to other organs, has been proposed in the pathogenesis of systemic sporotrichosis $[1-6,8,9]$. However, cutaneous inoculation can also lead to involvement of underlying tendons and joints, and can rarely disseminate $[1-3,6,7]$.

Therapy of sporotrichosis is primarily pharmacologic, but surgical intervention can play an important role, with resection occasionally being curative $[4,5,10]$. Spontaneous remissions of both cutaneous and pulmonary infections have rarely been reported [11-13]. However, due to the associated morbidity and rarely mortality, treatment is recommended for active infection $[5,8]$. In addition to difficulties with tolerance, toxicity, and administration, current regimens, including amphotericin $\mathrm{B}$, ketoconazole, or a saturated solution of potassium iodide (SSKI), are of limited efficacy in more recalcitrant 
systemic sporotrichosis. Therapeutic options of equal or greater efficacy are needed.

Clinical experience with the new oral triazole antifungal itraconazole continues to accumulate in the United States and worldwide. It has been used investigationally for several years in a variety of mycoses, with numerous reports supporting its safety and efficacy. Gastrointestinal and endocrine toxicities appear to be less frequent for itraconazole than for ketoconazole [14-16]. In vitro, itraconazole is active against $S$. schenckii, and animal models support its efficacy in the treatment of experimental sporotrichosis $[14,17,18]$. Relatively few reports have been published using itraconazole to treat sporotrichosis in humans, but these suggest therapeutic efficacy $[6,14,18,20,22]$. The highly lipophilic character with accumulation in the skin and its slow clearance make itraconazole an ideal agent for the treatment of sporotrichosis [14,17]. This report reviews our experience with itraconazole in the treatment of cutaneous inoculation and systemic sporotrichosis.

\section{PATIENTS AND METHODS}

Patients were treated either as part of a National Institute of Allergy and Infectious Diseases Mycoses Study Group open trial of itraconazole for endemic mycoses or on a compassionate treatment protocol made possible by Janssen Research Foundation. Patients were treated at 11 different institutions from 1984 to 1991 . All patients gave informed consent for treatment with itraconazole. Two patients in the current series were reported previously, but additional follow-up is included in this report $[6,15]$.

\section{Entry Criteria}

Patients with both cutaneous inoculation and systemic sporotrichosis were included. A culture positive for S. schenckii or histopathology compatible with this infection was required for inclusion in the study. If the patient had relapsed after prior therapy, a positive culture or clinical evidence of relapse was required.

\section{Treatment Regimens}

The dose of itraconazole ranged from 100 to 600 $\mathrm{mg}$ daily. Lower doses of itraconazole were used during early periods of this study, whereas doses of $400 \mathrm{mg}$ daily were commonly used during later periods; only one patient received $600 \mathrm{mg}$ daily. Dose escalation was allowed if the response was determined to be suboptimal. Patients were recommended to take their dose with food. Patients were evaluable if they had received at least a 3-month duration of itraconazole. One patient, who was lost to follow-up with active disease, was excluded from analysis based on this 3-month criterion. Concurrent therapy with other antifungal or immunologic agents was not allowed. Patients were not allowed to take concomitant antacids or $\mathrm{H}_{2}$ blockers because of the possibility of impaired absorption of itraconazole. Serum levels for itraconazole were not measured routinely. Pill counts to assess compliance were not performed.

\section{Response to Therapy}

Based on the investigators' assessment of outcome to treatment with itraconazole, patients were classified as responders or nonresponders. Responders were further classified as remaining on treatment, relapsed, free of disease, or lost to follow-up. Responders remaining on treatment were determined by investigators to have evidence of improvement. Relapse was determined by the development of new or recurrent lesions after itraconazole was discontinued. Nonresponders included those patients who failed to respond or progressed during treatment with itraconazole. Both nonresponders and responders who relapsed were considered treatment failures. However, responders who relapsed could receive a second course of itraconazole, and the outcome of this course was classified separately.

\section{RESULTS}

\section{Patient Characteristics}

Twenty-seven patients with sporotrichosis were treated with 30 courses of itraconazole. Demographic features and risk factors are presented in Table I for the overall patient cohort and for each of the four disease categories (lymphocutaneous, systemic unifocal pulmonary, systemic unifocal articular/osseous, and systemic multifocal sporotrichosis). Twenty-one patients were white, and two each were black, Native American, and Hispanic. Of the six nonwhite patients, five had systemic involvement. There were 22 men and 5 women, with a mean age of 53 years and a range of 22 to 86 years. Alcoholism and diabetes mellitus were common underlying illnesses, present in seven and eight patients, respectively. One patient with pulmonary sporotrichosis had underlying chronic obstructive pulmonary disease (COPD). Of the 16 patients with underlying illnesses, 14 had systemic involvement. No patient had severe immunosuppression. Four patients had hobbies or occupations that placed them at risk for acquiring sporotrichosis. One of these patients acquired lymphocutaneous disease following inoculation from a laboratory accident. Another patient with lymphocutaneous involvement was a Christmas tree farmer. One patient, with pulmonary involvement, operated heavy construction equipment in landscaping and earthmov- 


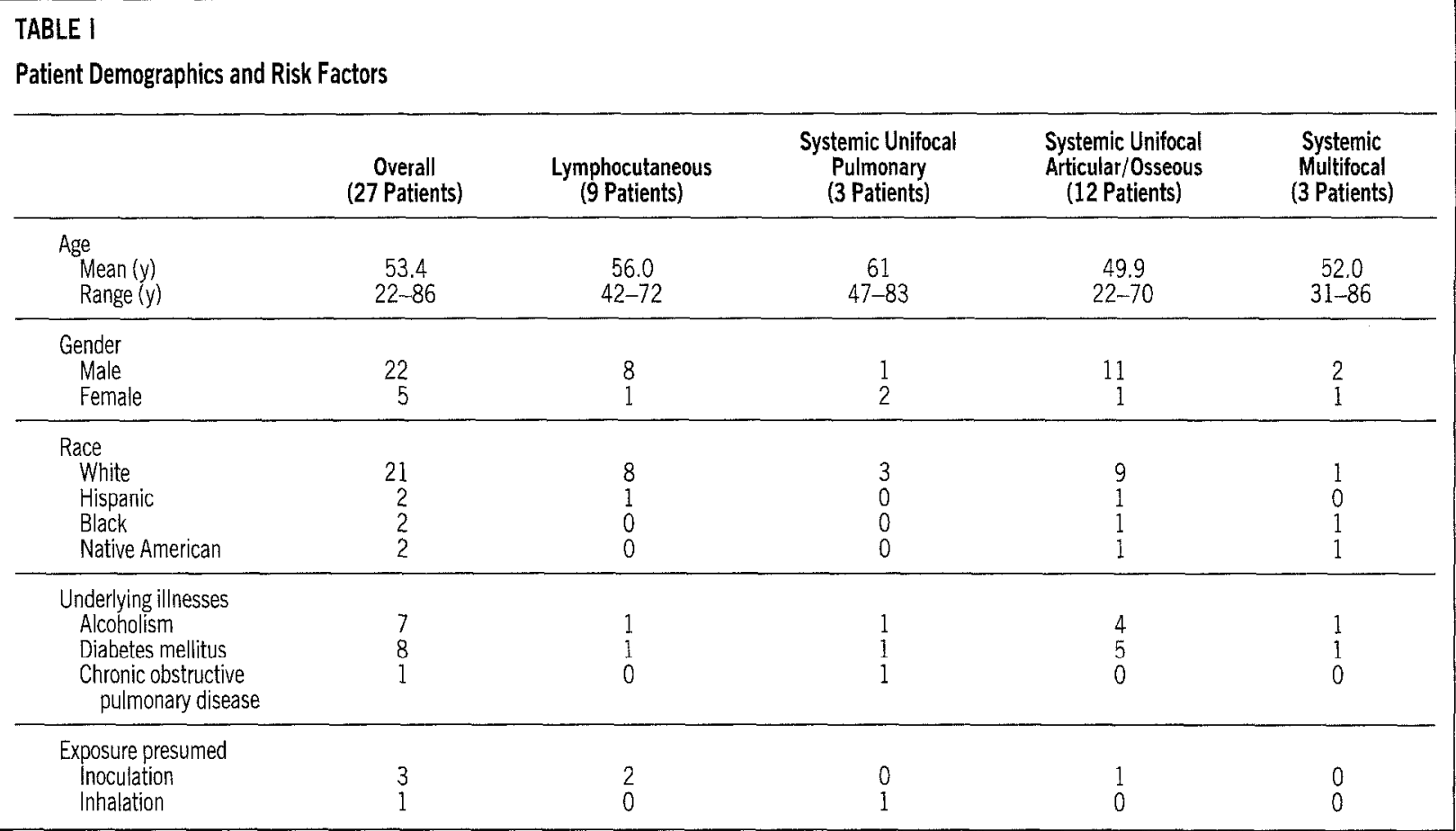

ing. The fourth patient, with articular/osseous involvement, was a rose gardener.

\section{Sites of Involvement}

Sites of involvement included lymphocutaneous in 9 patients, articular/osseous in 15 ( 3 with multifocal), and lung in 3 (unifocal). Seven of nine patients with lymphocutaneous disease had skin lesions only, and two had skin lesions and lymph node involvement. Eleven of 15 patients with articular/osseous sporotrichosis had arthritis, with or without contiguous bone involvement, 2 had tenosynovitis, and 2 had osseous sporotrichosis without joint involvement. Twelve patients had unifocal articular/osseous involvement. Of the three patients with systemic multifocal sporotrichosis, two had two joints involved, and one had a single joint involved and a distant cutaneous lesion. The knee, in seven patients, was the most common joint involved, followed by the wrist and elbow in four and three patients, respectively. Excluding the two patients with more than one joint involved, the right side was involved in all five patients with upper extremity disease. Central nervous system involvement was not observed.

All but one patient had a positive culture for $S$. schenckii at the time that treatment with itraconazole commenced. However, this patient had a positive culture 2 months into treatment with itraconazole. One patient, relapsing after his initial course of itraconazole, had a positive culture prior to a course of fluconazole during which disease progressed, but the culture was not repeated prior to the second course of itraconazole. Histopathology was compatible with sporotrichosis in 11 patients.

\section{Symptoms and Signs}

The only constitutional symptom reported was weight loss in one patient with pulmonary sporotrichosis. Fever was not reported. Patients with lymphocutaneous involvement typically had the skin lesion and/or lymphadenopathy as the only symptom and sign reported. Erythema was described in two patients, pain and ulceration in one patient, and drainage in one patient with lymphocutaneous sporotrichosis. One patient with articular involvement had no symptoms or signs recorded, but all other patients with articular/osseous sporotrichosis had pain and swelling of the affected joint. Cutaneous drainage was described in four patients, decreased range of motion in three patients, and increased warmth over the affected area in three patients with articular/osseous sporotrichosis. All three patients with pulmonary sporotrichosis had cough and sputum production. Hemoptysis was noted in two patients, and chest pain in one patient with pulmonary sporotrichosis.

\section{Prior Therapy}

Eleven patients had received prior unsuccessful therapy for sporotrichosis. The manner in which prior therapy was recorded varied among investigators. Some patients received more than one agent. Therapy with SSKI was attempted in four patients, three with lymphocutaneous and one with articular/osseous involvement. SSKI was discontinued in 


\section{TABLE \|}

Outcome of Treatment With Itraconazole in 30 Episodes of Sporotrichosis in 27 Patients

\begin{tabular}{lccccccc}
\hline $\begin{array}{c}\text { Type of } \\
\text { Response }\end{array}$ & $\begin{array}{c}\text { No. of } \\
\text { Courses }\end{array}$ & \multicolumn{2}{c}{$\begin{array}{c}\text { ITZ Dose } \\
\text { (mg/d) }\end{array}$} & & \multicolumn{2}{c}{$\begin{array}{c}\text { ITZ Duration } \\
\text { (mo) }\end{array}$} \\
\hline Nonresponder & 5 & 380 & Range & & Mean & Range \\
\hline Responder & 25 & & & & & \\
$\quad$ Remain on ITZ & 3 & 367 & $300-400$ & & $10.5+$ & $6-18$ \\
Relapsed & 7 & 333 & $200-400$ & 7.4 & $5-18$ \\
Lost to F/U & 1 & 400 & N/A & 10 & $6-18$ \\
Free of disease & 14 & 250 & $100-400$ & 7.8 & $3-15$ \\
\hline
\end{tabular}

ITZ = itraconazole; $F / U=$ follow-up; N/A = not applicable.

one patient because of toxicity after 5 days, and in three for failure after 12 days, 3 months, and 5 months, respectively. Four patients with articular/osseous sporotrichosis and one with pulmonary involvement received therapy with amphotericin $B$, at total doses of 1.5 to $2.5 \mathrm{~g}$ (mean: $2.0 \mathrm{~g}$ ); one failed to respond and the other four relapsed after therapy was stopped. Therapy with ketoconazole failed in six patients, and with fluconazole in two others. Two of the six patients received less than 1 month of treatment with ketoconazole at $400 \mathrm{mg} / \mathrm{d}$. Total doses of 24 and $36 \mathrm{~g}$ of ketoconazole were recorded in two other patients. Another patient failed separate courses of 7 and 9 months' duration at $400 \mathrm{mg}$ daily of ketoconazole. The other patient in whom ketoconazole failed received more than 4 years of therapy with doses up to $600 \mathrm{mg}$ daily.

\section{Response to Itraconazole Therapy}

Itraconazole therapy was given at doses of 100 to $600 \mathrm{mg}$ daily over durations that ranged from 3 to 18 months, with three patients continuing on treatment. Most patients received 200 or $400 \mathrm{mg}$ daily. Three patients received a second course of therapy with itraconazole and are counted twice in the analysis of outcomes. The overall response to itraconazole treatment is presented in Table II and responses in each of the four different categories of disease in Table III.

OVERALL RESPONSE TO ITRACONAZOLE: There were 25 responders and 5 nonresponders to the 30 courses of itraconazole. All nonresponders received at least $200 \mathrm{mg}$ daily, and one received $600 \mathrm{mg}$ daily, over durations ranging from 6 to 18 months. Of the 25 responders, 7 relapsed 1 to 7 months after treatment durations ranging from 6 to 18 months. Three of these seven responders who relapsed were treated with a second course of itraconazole. One patient again responded to a 6 -month course of itraconazole but experienced a second relapse (included twice in the 25 responders and twice in the 7 relapses). The 2 other patients appear to be responding to their sec-
TABLE III

Outcome of Treatment With Itraconazole by Disease Category

\begin{tabular}{|c|c|c|c|c|c|}
\hline \multirow{2}{*}{$\begin{array}{c}\text { Type of } \\
\text { Response }\end{array}$} & \multirow{2}{*}{$\begin{array}{l}\text { No. of } \\
\text { Courses }\end{array}$} & \multicolumn{2}{|c|}{$\begin{array}{l}\text { ITZ Dose } \\
\text { (mg/d) }\end{array}$} & \multicolumn{2}{|c|}{$\begin{array}{l}\text { ITZ Duration } \\
\text { (mo) }\end{array}$} \\
\hline & & Mean & Range & $\overline{\text { Mean }}$ & Range \\
\hline \multicolumn{6}{|l|}{ Lymphocutaneous } \\
\hline \multirow{2}{*}{\multicolumn{6}{|c|}{$\begin{array}{l}\text { Nonresponder } \\
\text { Responder }\end{array}$}} \\
\hline & & & & & \\
\hline Remain on ITZ & 1 & 300 & N/A & $7.5+$ & N/A \\
\hline Relapsed & 2 & 200 & $N / A$ & 5.5 & $5-6$ \\
\hline Free of disease & 7 & 228 & $100-400$ & 6.3 & $3-12$ \\
\hline \multicolumn{6}{|l|}{ Systemic unifocal-- } \\
\hline \multirow{2}{*}{\multicolumn{6}{|c|}{$\begin{array}{l}\text { Nonresponder } \\
\text { Responder }\end{array}$}} \\
\hline & & & & & \\
\hline \multirow{3}{*}{$\begin{array}{l}\text { Remain on ITZ } \\
\text { Relapsed } \\
\text { Free of disease }\end{array}$} & 1 & 400 & $N / A$ & $18+$ & N/A \\
\hline & 1 & 400 & $N / A$ & 18 & N/A \\
\hline & 1 & 200 & $N / A$ & 12 & $\mathrm{~N} / \mathrm{A}$ \\
\hline \multicolumn{6}{|l|}{ Systemic unifocal- } \\
\hline \multicolumn{6}{|l|}{ Nonresponder } \\
\hline \multicolumn{6}{|l|}{ Responder } \\
\hline \multirow{4}{*}{$\begin{array}{l}\text { Remain on ITZ } \\
\text { Relapse } \\
\text { Lost to F/U } \\
\text { Free of disease }\end{array}$} & 1 & 400 & $N / A$ & 6 & $N / A$ \\
\hline & 4 & 350 & $200-400$ & 5.8 & $5-6$ \\
\hline & 1 & 400 & & 10 & $\mathrm{~N} / \mathrm{A}$ \\
\hline & 4 & 245 & $200-300$ & 7.8 & $6-12$ \\
\hline \multicolumn{6}{|l|}{ Systemic multifocal } \\
\hline \multirow{2}{*}{$\begin{array}{l}\text { Nonresponder } \\
\text { Resposider } \\
\text { Free of disease }\end{array}$} & 1 & 400 & $\mathrm{~N} / \mathrm{A}$ & 6 & $\mathrm{~N} / \mathrm{A}$ \\
\hline & $\begin{array}{l}2 \\
2\end{array}$ & 400 & $N / A$ & 11 & $7-15$ \\
\hline
\end{tabular}

ITZ = itraconazole; F/U = follow-up; $N / A=$ not applicable

ond course of treatment (each included twice in the 25 responders and once in the 7 relapses). One responder completing 10 months of treatment with itraconazole was lost to follow-up. Of the remaining 17 responders, 3 remain on treatment (including the 2 treated previously with itraconazole but relapsing), and 14 remain free of disease. Of these 14 responders who remain free of disease, doses ranged from 100 to $300 \mathrm{mg}$ daily (mean: $250 \mathrm{mg}$ ), and follow-up duration ranged from 6 to 42 months (mean: 17.6 months). The other responding patient, still remaining on his initial treatment course, has resolved lesions on itraconazole.

RESPONSE OF LYMPHOCUTANEOUS SPOROTRICHOSIS TO ITRACONAZOLE: Of the 10 courses of treatment in the 9 patients with lymphocutaneous sporotrichosis, all patients were responders, although 1 patient relapsed after both courses of itraconazole (counted twice as a responder who relapsed). One patient remains on his initial course of itraconazole (7.5 months) with resolution of lesions that had failed to respond to 5 months of treatment with SSKI. The duration of follow-up in the seven responders who remained free of disease ranged from 6 to 42 months (mean: 17.4 months).

RESPONSE OF PULMONARY SPOROTRICHOSIS TO ITRACONAZOLE: Two patients with unifocal pulmonary sporotrichosis had failed prior treatment. One patient failed to respond to itraconazole at a dose of $300 \mathrm{mg}$ daily for 9 months. The other patient re- 
ceived two courses of itraconazole. Despite resolution of symptoms and improvement in radiographic abnormalities, this patient relapsed after his initial 18-month course of itraconazole at $400 \mathrm{mg}$ daily. Symptoms have improved during the second course at the same dose of itraconazole. The patient continues on this treatment after 18 months and is considered a responder. However, sputum cultures still yielded S. schenckii after 12 months. The third patient with pulmonary sporotrichosis received 11.5 months of itraconazole (maximum dose of 200 $\mathrm{mg}$ daily) and remains free of disease 40 months after discontinuation.

RESPONSE OF ARTICULAR/OSSEOUS SPOROTRICHOSIS TO ITRACONAZOLE: Twelve of the 15 patients with articular/osseous sporotrichosis had unifocal disease. Of the 13 courses of treatment in those with unifocal disease, 10 were responders, and 3 were nonresponders. Four responders relapsed 1 to 6 months after itraconazole courses of only 5 or 6 months at 200 to $400 \mathrm{mg}$ daily. One of these patients continues on a second course of itraconazole at 400 $\mathrm{mg}$ daily and is considered a responder at 6 months (included twice as a responder but only once as a relapse). One patient treated for 10 months was considered a responder but was lost to follow-up. The other four responders remain free of disease with follow-up durations of 12 to 18 months (mean: 16.5 months). These four patients received itraconazole doses of 200 to $300 \mathrm{mg}$ daily over durations ranging from 6 to 12 months (mean: 7.8 months). Of the three patients with multifocal disease, one was a nonresponder, and two remain free of disease 6.5 and 18 months after treatment ended.

RESPONSE TO ITR ACONAZOLE IN PATIENTS WITH UNDERLYING ALCOHOLISM AND DIABETES MELLITUS: Among the seven patients with underlying alcoholism, there were five responders and two nonresponders. Of the five responders, one was lost to follow-up, and four remained free of disease, only one of whom had lymphocutaneous sporotrichosis. Among the eight patients with underlying diabetes mellitus, there were seven responders and one nonresponder. Of the seven responders, three relapsed, and four remained free of disease, only one of whom had lymphocutaneous sporotrichosis.

\section{Adverse Effects}

Twelve patients experienced adverse effects possibly related to itraconazole treatment. Four patients experienced nausea, and one also had vomiting. One of these patients also had malaise, arthralgias, and mood disturbances during the treatment course, but the relationship of these to itraconazole was unclear. Doses in these four patients were 100 to $400 \mathrm{mg}$ daily. One patient experienced a rise in serum transaminase levels to twice normal limits. Itraconazole treatment was held for 2 weeks in this patient and then resumed without additional adverse effects. One patient experienced an elevation in the serum bilirubin concentration to $2.4 \mathrm{mg} / \mathrm{dL} 1 \mathrm{month}$ after itraconazole doses were raised to $600 \mathrm{mg}$ daily, which resolved upon discontinuation of itraconazole. One patient experienced transient nervousness.

Two patients experienced the onset of mild hypertension during treatment with itraconazole. Both were receiving doses of $400 \mathrm{mg}$ daily. One also experienced peripheral edema coincident with hypertension. One other patient also developed peripheral edema during treatment. Due to underlying coronary artery disease and chronic renal failure in this patient, the relationship to itraconazole was uncertain.

Two patients experienced possible adverse drug interactions while receiving itraconazole therapy. One patient receiving concurrent warfarin therapy experienced an increase in the prothrombin time. Another patient experienced a rise in serum digoxin levels requiring dosage reduction of digoxin.

\section{COMMENTS}

The male predominance in this series, as well as in most other series of sporotrichosis, is attributed to outdoor occupations and hobbies [2,5,6,23]. A higher prevalence in women has been reported from Japan [24]. Only three of our patients had infection readily attributed to hobbies or outdoor occupational exposure. There appears to be no racial predilection; whites represent the majority of patients in the current series and other reports [5]. However, five of the six nonwhite patients in the current series had systemic disease. The possible influence of race on the presentation and course of sporotrichosis deserves further study.

Alcoholism has been recognized as a prominent risk factor in systemic sporotrichosis $[2,4-6,8]$. Of seven patients with alcoholism, five had articular/osseous sporotrichosis. Other diseases reported as potential risk factors and noted in our patients include diabetes mellitus and COPD; eight of nine patients with these diseases had systemic sporotrichosis. Immunosuppressive conditions, such as corticosteroid therapy, hematologic malignancy, transplantation, or human immunodeficiency virus infection, were not seen in our patients [5-8,24-26].

Pulmonary sporotrichosis, once thought rare, has been increasingly recognized [2]. Cavitary upper lobe lesions are characteristic of unifocal systemic pulmonary sporotrichosis $[2-5,27]$. This presentation is easily confused with reactivation pulmonary tuberculosis. In contrast, pulmonary involvement in multifocal systemic disease, noted in approximately $20 \%$ of patients, is characterized by linear and nodular infiltrates [2]. 
Articular/osseous sporotrichosis is most often thought to follow hematogenous dissemination rather than inoculation with contiguous invasion $[2,6,8,9,23]$. Typical radiographic abnormalities include erosions of the articular surfaces with lytic lesions of the surrounding bone $[2,8,23]$. The lower extremity is most frequently involved [6,23]. Interestingly, all of our patients with unifocal articular/osseous sporotrichosis of the upper extremity had involvement on the right. Assuming hematogenous dissemination as the underlying pathogenesis, one would expect an equal distribution between the right and left upper extremities as well as involvement of the axial skeleton. Thus, there may be a role for local inoculation in the pathogenesis of some cases of articular/osseous sporotrichosis.

Cutaneous inoculation sporotrichosis usually responds to SSKI $[4,5,13,18,20]$. Although SSKI is inexpensive, it is frequently not well tolerated [18]. Allergic reactions and gastrointestinal intolerance are common $[4,18]$. Systemic sporotrichosis is usually resistant to treatment with iodides and requires therapy with amphotericin B $[2,4-6,8,10,20,23]$. However, some patients fail to respond, and relapses do occur $[4,5,7,10]$. In addition, amphotericin $\mathrm{B}$ has significant toxicity and inherent difficulties associated with intravenous administration [14]. Intra-articular administration of amphotericin B has been successful in a few patients with articular involvement [28]. Surgical intervention combined with iodides or amphotericin B increases the efficacy of treatment of pulmonary and articular/osseous sporotrichosis $[2,4,5,8,10,23]$.

Successful treatment with intravenous miconazole in pulmonary sporotrichosis resistant to amphotericin B has been reported [29]. However, miconazole has failed in other cases and also has associated toxicities $[4,5,14,29]$. Treatment with ketoconazole has been associated with variable results. Although an initial response may occur, relapses are not infrequent $[4,5,27,30-34]$. One series reported a better response rate when doses of 400 to $800 \mathrm{mg}$ daily were used [31]. Many of these patients had concurrent surgical débridement, and the majority were treated for more than 1 year [31]. The authors suggested that the high proportion of sustained remissions they observed may have related to the longer duration of treatment [31]. Unfortunately, at these higher doses, a greater incidence and severity of adverse effects, including gastrointestinal and endocrine effects, are noted $[14,30,35,36]$.

The primary mechanism of the action of itraconazole, as with other azoles, is inhibition of ergosterol biosynthesis. In vitro studies have demonstrated the minimal inhibitory concentration (MIC) of itraconazole for S. schenckii (yeast phase) to be from 0.1 to $12.5 \mathrm{mg} / \mathrm{L}$, similar to the MIC of 0.1 to 10 $\mathrm{mg} / \mathrm{L}$ for ketoconazole $[17,18,21,31]$.
Efficacy with itraconazole, at $100 \mathrm{mg}$ daily, was demonstrated in the treatment of lymphocutaneous sporotrichosis in a series from South America. Of 17 patients treated for a mean duration of 130 days, 14 remained free of relapse with an average follow-up of 115 days [18]. A report of five patients from Mexico also supported the efficacy of itraconazole at doses of 100 to $200 \mathrm{mg}$ daily [20]. In patients with lymphocutaneous sporotrichosis in this series, we found a similar response rate. All nine patients responded, one remains on treatment, and seven remain free of disease after discontinuation of itraconazole over a mean follow-up duration of 17 months. Relapse occurred in only one patient, to two separate courses.

The treatment of pulmonary sporotrichosis is problematic $[4,5,10,27]$. Cure rates of unifocal pulmonary sporotrichosis treated with amphotericin B are less than $50 \%[4,5,10]$. Cure rates as high as $70 \%$ to $80 \%$ have been achieved with a combination of surgical resection and amphotericin $B$ or SSKI. However, resection is not always feasible, especially in patients with underlying COPD $[4,5,10]$. Outcomes with miconazole and ketoconazole have failed to document their efficacy $[5,27,29,30]$. With only three patients with pulmonary sporotrichosis, it is difficult to compare our treatment outcomes with itraconazole to those of other therapies, but it appears to be no better than noted previously.

Articular/osseous sporotrichosis has also been very difficult to treat. SSKI is ineffective $[1,2,6,8,10,21]$. At least $30 \%$ to $40 \%$ of patients treated with amphotericin B fail therapy or relapse $[10,23,28]$. In successful cases, a total dose of 1 to $5 \mathrm{~g}$ of amphotericin $\mathrm{B}$ has been required $[1,2,6,8,10,23]$. Treatment outcomes with ketoconazole, for articular/osseous sporotrichosis, have been variable [30-33]. Some authors have suggested that higher doses and longer durations may result in more favorable outcomes and sustained remissions $[31,33]$. In the 15 patients with articular/osseous sporotrichosis in the current series, itraconazole treatment outcomes were comparable to those reported with other therapies. Eleven of the 15 patients were considered responders. However, 4 of these 11 relapsed 1 to 6 months later, and no follow-up is available in 1. All four patients relapsing had received only 5 to 6 months of treatment. The other six patients, including two with multifocal involvement, remain free of disease over a mean follow-up duration of 15 months.

The results in the current series of patients with sporotrichosis support the efficacy of itraconazole. Favorable results were obtained in both cutaneous inoculation and systemic sporotrichosis. In the 18 patients with systemic sporotrichosis in this series, the treatment outcomes with itraconazole are com- 
parable to results with amphotericin B and ketoconazole. Unfortunately, no pill counts or measurement of serum levels were done to assess itraconazole compliance.

Itraconazole therapy appears to have less toxicity than either ketoconazole or amphotericin B. The occurrence of hypertension, which was seen in two patients treated with $400 \mathrm{mg}$ daily, has been noted previously [16]. This side effect appears to respond to conservative medical management and should not interfere with therapy [16]. Itraconazole represents a reasonable alternative to amphotericin $B$ and may be more efficacious than ketoconazole in the treatment of systemic sporotrichosis. In the treatment of lymphocutaneous sporotrichosis, itraconazole represents a reasonable alternative to SSKI. It may be better tolerated than SSKI, and it may offer a less toxic alternative over either ketoconazole or amphotericin $B$. Itraconazole should be a very useful addition to the antifungal armamentarium for treatment of sporotrichosis.

\section{APPENDIX}

\section{Other Members of the NIAID Mycoses Study Group}

John Bennett, M.D., National Institute of Allergy and Infectious Diseases, Bethesda, MD; Robert W. Bradsher, M.D., University of Arkansas for Medical Sciences, Little Rock, AR; Stanley W. Chapman, M.D., University of Mississippi Medical Center, Jackson, MS; John F. Fisher, M.D., Medical College of Georgia, Augusta, GA; Thomas M. Kerkering, M.D., Medical College of Virginia, Richmond, VA; Gerald Medoff, M.D., Washington University School of Medicine, St. Louis, MO; John R. Perfect, M.D., Duke University Medical Center, Durham, NC; George A. Pankey, M.D., Ochsner Clinic, New Orleans, LA; J.H. Rex, M.D., National Institute of Allergy and Infectious Diseases, Bethesda, MD; Michael S. Saag, M.D., University of Alabama at Birmingham, Birmingham, AL.

\section{REFERENCES}

1. Wilson DE, Mann JJ, Bennett JE, Utz JP. Clinical features of extracutaneous sporotrichosis. Medicine (Baltimore) 1967; 46: 265-79.

2. Lynch PJ, Voorhees JJ, Harrell ER. Systemic sporotrichosis. Ann Intern Med 1970; 73: 23-30.

3. Watts JC, Chandier FW. Primary pulmonary sporotrichosis. Arch Pathol Lab Med 1987; 111: 215-7.

4. Gerding DN. Treatment of pulmonary sporotrichosis. Semin Respir Infect 1986; 1: 61-5.

5. Pluss JL, Opal SM. Pulmonary sporotrichosis: review of trcatment and out come. Medicine (Baltimore) 1986; 65: 143-53.

6. Lesperance $M$, Baumgartner D, Kauffman CA. Polyarticular arthritis due to Sporothrix schenckii. Mycoses 1988; 31: 599-603.

7. Gullberg RM, Quintanilla A, Levin ML, Williams J, Phair JP. Sporotrichosis: recurrent cutaneous, articular, and central nervous system infection in a renal transplant recipient. Rev Infect Dis 1987; 9: 369-75.

8. Crout JE, Brewer NS, Tompkins RB. Sporotrichosis arthritis: clinical features in seven patients. Ann Intern Med 1977; 86: 294-7.
9. Brook CJ, Ravikrishnan KP, Weg JG. Pulmonary and articular sporotrichosis. Am Rev Respir Dis 1977; 116: 141-3.

10. Parker JD, Sarosi GA, Tosh FE. Treatment of extracutaneous sporotrichasis. Arch Intern Med 1970; 125: 858-63.

11. Pueringer RJ, Iber C, Deike MA, Davies SF. Spontaneous remission of extensive pulmonary sporotrichosis. Ann Intern Med 1986; 104: 366-7.

12. England DM, Hochholzer L. Sporothrix infection of the lung without cutaneous disease. Arch Pathol Lab Med 1987; 111: 298-300.

13. Iwatsu T, Nishimura K, Miyaji M. Spontaneous disappearance of cutaneous sporotrichosis: report of two cases. Dermatology 1985; $24: 524-5$.

14. Saag MS, Dismukes WE. Azole antifungal agents: emphasis on new triazoles. Antimicrob Agents Chemother 1988; 32: 1-8.

15. Phillips P, Graybill JR, Fetchick R, Dunn JF. Adrenal response to corticotropin during therapy with itraconazole. Antimicrob Agents Chemother 1987; 31: 647-9. 16. Sharkey PK, Rinaldi MG, Dunn JF, Hardin TC, Fetchick RJ, Graybill JR. High-dosc itraconazole in the treatment of scvere mycoscs. Antimicrob Agents Chemother 1991; 35: 707-13.

17. Van Cutsem J, Van Gerven F, Janssen PAJ. Activity of orally, topically, and parenterally administered itraconazole in the treatment of superficial and deep mycoses: animal models. Rev Infect Dis 1987; 9 Suppl: S15-32.

18. Restrepo A, Robledo J, Gomez I, Tabares AM, Gutierrez R. Itraconazole therapy in lymphangitic and cutaneous sporotrichosis. Arch Dermatol 1986; 122: 413-7.

19. Hardin TC, Graybill JR, Fetchick R, Woestenborghs R, Rinaldi MG, Kuhn JG. Pharmacokinetics of itraconazole following oral administration to normal volunteers. Antimicrob Agents Chemother 1988; 32: 1310-3.

20. Lavalle P, Suchil P, De Ovando F, Reynoso S. Itraconazole for deep mycoses: preliminary experience in Mexico. Rev Infect Dis 1987; 9 Suppl: S64-70. 21. Tucker RM, Williams PL, Arathoon EG, Stevens DA. Treatment of mycoses with itraconazole. Annals N Y Acad Sci 1988; 544: 451-70.

22. Albanese G, Dicintio R, Sala G, Galblati G. Itraconazole in the treatment of sporotrichosis. J de Mycologie Medicale (Pasteur Institue) 1992; 2: 115-6. 23. Gladstone $\mathrm{JL}$, Littman $\mathrm{ML}$. Osseous sporotrichosis: failure of treatment with potassium iodide and sulfadimethoxine and success with amphotericin B. Am J Med 1971; 51: 121-33.

24. Kusuhara M, Hachisuka H, Sasai Y. Statistical survey of 150 cases with sporotrichosis. Mycopathologia 1988; 102: 129-33.

25. Bibler MR, Luber HJ, Glueck HI, Estes SA. Disseminated sporotrichosis in a patient with HIV infection after treatment for acquired factor VIII inhibitor. JAMA 1986; 256: 3125-6.

26. Kurosawa A, Pollack SC, Collins MP, Kraff CR, Tso MOM. Sporothrix schenckii endophthalmitis in a patient with human Immunodeficlency virus infection. Arch Ophthalmol 1988; 106: 376-80.

27. Dall L, Salzman G. Treatment of pulmonary sporotrichosis with ketoconazole. Rev Infect Dis 1987; 9: 795-8.

28. Downs NJ, Hinthorn DR, Mhatre VR, Liu C. Intra-articular amphotericin $B$ treatment of Sporothrix schenckii arthritis. Arch Intern Med 1989; 149: 954-5.

29. Rohwedder JJ, Archer G. Pulmonary sporotrichosis: treatment with miconazole. Am Rev Respir Dis 1976; 114: 403-6.

30. Dismukes WE, Stamm AM, Graybill JR, et al. Treatment of systemic mycoses with ketoconazole: emphasis on toxicity and clinical response in $52 \mathrm{pa}-$ tients. Ann Intern Med 1983; 98: 13-20.

31. Calhoun DL, Waskin H, White MP, et al. Treatment of systemic sporotrichosis with ketoconazole. Rev Infect Dis 1991; 13: 47-51.

32. Horsburgh CR Jr, Cannady PB Jr, Kirkpatrick $\mathrm{CH}$. Treatment of fungal infections in the bones and joints with ketoconazole. J Infect Dis 1983; 147: 1064-9.

33. Graybill JR, Craven PC, Donovan W, Matthew EB. Ketoconazole therapy of systemic fungal infections. Inadequacy of standard dosage regimens. Am Rev Respir Dis 1982; 126: 171-4.

34. Symoens J, Moens M, Dom J, et al. An evaluation of two years of clinical experience with ketoconazole. Rev Infect Dis 1980; 2: 674-87.

35. Sugar AM, Alsip SG, Galgiani JN, et al. Pharmacology and toxicity of highdose ketoconazole. Antimicrob Agents Chemother 1987; 31: 1874-8.

36. Pont A, Graybill JR, Craven PC, et al. High-dose ketoconazole therapy and adrenal and testicular function in humans. Arch Intern Med 1984; 144: 2150-3. 